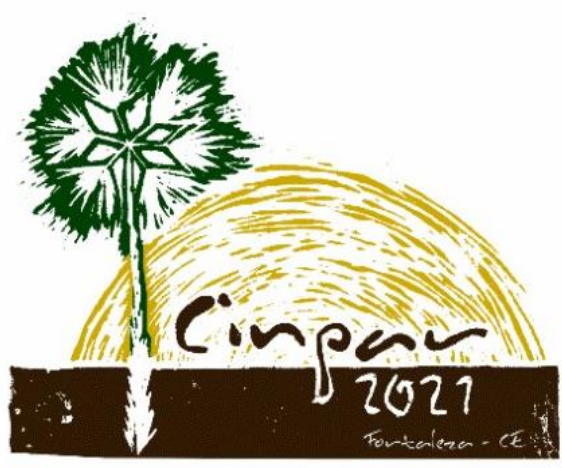

XVII Congresso Internacional sobre Patologia e

Reabilitação das Construções

XVII Congreso Internacional sobre Patología y Rehabilitación de las Construcciones

XVII International Conference on Pathology and Constructions Rehabilitation

FORTALEZA (Brasil), 3 a 5 de junho de 2021 https://doi.org/10.4322/CINPAR.2021.024

\title{
Influência da resistência à compressão do substrato de concreto no descolamento interfacial de sistemas PRFC laminados in situ.
}

\section{The influence of concrete substrate compressive strength on the interfacial debonding of wet layup CFRP systems.}

\author{
Mônica Regina GARCEZ ${ }^{1}$, Abrahão Bernardo ROHDEN² \\ ${ }^{1}$ Universidade Federal do Rio Grande do Sul, Porto Alegre, Brasil, monica.garcez@ufrgs.br \\ ${ }^{2}$ Universidade Regional de Blumenau, Blumenau, Brasil, arohden@furb.br
}

\begin{abstract}
Resumo
Este trabalho investiga a influência da resistência à compressão do substrato de concreto no descolamento interfacial de sistemas PRFC laminados in situ. Foram realizados ensaios de flexão a quatro pontos em vigas de concreto armado de escala reduzida, projetados para simular tensões normais e de cisalhamento induzidas na interface entre o PRFC e o concreto. As vigas, de $150 \mathrm{~mm} \times 150 \mathrm{~mm} \times 600 \mathrm{~mm}$ e armadas com duas barras de aço de $5 \mathrm{~mm}$ e estribos de aço de $6,3 \mathrm{~mm}$ espaçados de $75 \mathrm{~mm}$, foram reforçadas com uma camada de PFRC colado ao substrato de concreto de $30 \mathrm{MPa}, 50 \mathrm{MPa}, 80 \mathrm{MPa}$ ou $90 \mathrm{MPa}$. Os mecanismos de iniciação do dano e propagação do descolamento mostraram-se altamente dependentes da transferência de carga e da redistribuição de tensões após a fissuração do concreto. A resistência a compressão do concreto afetou a resposta pré-fissuração, a transferência de tensão e a ductilidade geral na ruptura, resultando em um esquema de reforço menos eficiente. Em condições de serviço, as deformações no PRFC foram menores quanto maior a resistência à compressão do concreto.
\end{abstract}

Palavras-chave: falha por descolamento interfacial, resistência à compressão do concreto, PRFC.

\begin{abstract}
This paper investigates the influence of concrete substrate compressive strength on the interfacial debonding of wet layup CFRP systems. The assessment was made through small scale beam tests designed to simulate

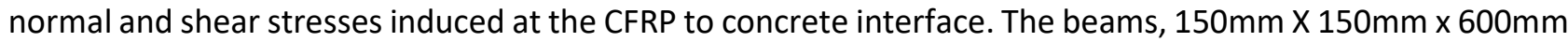
reinforced with $5 \mathrm{~mm}$ steel bars and $6.3 \mathrm{~mm}$ steel stirrups spaced $75 \mathrm{~mm}$ apart, were strengthened with one layer of CFRP bonded in a concrete substrate with compressive strength of $30 \mathrm{MPa}, 50 \mathrm{MPa}, 80 \mathrm{MPa}$, or $90 \mathrm{MPa}$. The mechanisms of debonding initiation and propagation are highly dependent on the load transferring and stress redistribution after concrete cracking. The concrete compressive strength affected the pre-cracking response, the stress transfer, and the overall ductility at failure, resulting in less FRP strengthening effectiveness. For service conditions, FRP strains were lower the higher the concrete compressive strength.
\end{abstract}

Keywords: interfacial debonding failure, concrete compressive strength, CFRP. 


\section{Introdução}

O desempenho de um reforço executado com Polímero Reforçado com Fibra (PRF) colado externamente a estruturas de concreto depende fundamentalmente da aderência entre o reforço e o substrato de concreto (Ulaga et al., 3003; Siddika et al., 2020). O sucesso da operaçao de reforço depende, portanto, do comportamento da interface entre o PRF e o concreto (Garcez et al., 2008; Dai et al., 2005). Os modos de ruptura mais comuns relacionados ao descolamento de sistemas PRF colados externamente a vigas de concreto armado são mostrados na Figura 1. Em (a) e (b) as rupturas de referem à propagação de fissuras intermediárias, de flexão ou cisalhamento. As rupturas mostradas em (d) e (e) estão relacionadas à capacidade insuficiente de ancoragem do elemento de reforço ou ao arrancamento do concreto (Triantafillou \& Matthys, 2010). A ruptura exemplificada em (c) pode ocorrer em vigas de concreto armado reforçadas ao cisalhamento ou com ancoragem adicional.

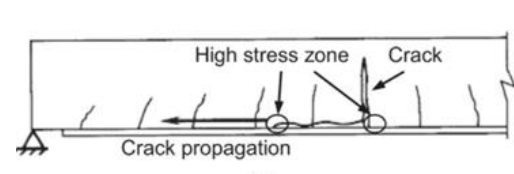

(a)

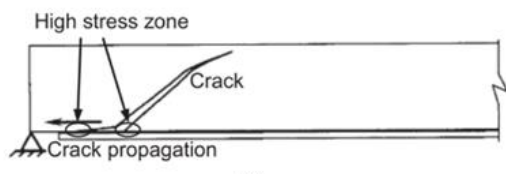

(b)

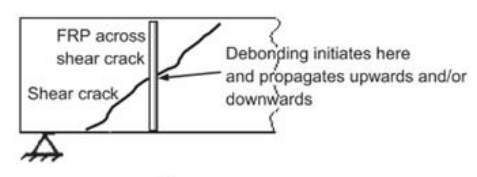

(c)

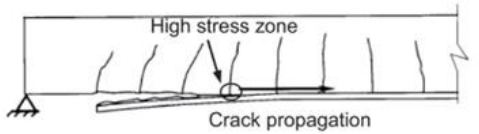

(d)

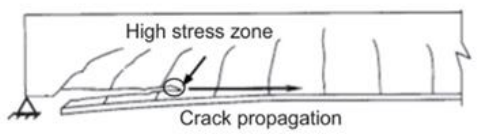

(e)

Figura 1 - Modos de ruptura por descolamento de sistemas PRF (Ullaga et al., 2003): (a) Propagação de fissura intermediária de flexão; (b) Propagação de fissura intermediária de cisalhamento; (c) Abertura de fissura de cisalhamento na ancoragem adicional; (d) Ancoragem insuficiente; (e) Arrancamento do concreto.

As recomendações dos códigos de projeto para evitar o descolamento do PRF ou o arrancamento do concreto tendem a limitar as deformações e tensões últimas no PRF com base nas propriedades mecânicas do substrato de concreto, na rigidez do sistema PRF e outros fatores obtidos a partir de dados experimentais (Triantafillou \& Matthys, 2010; ACl, 2017). As equações sugeridas nestes códigos são baseadas em modelos calibrados com resultados experimentais disponíveis na literatura. As variações e os graus de complexidade dos diferentes modelos, que refletem a complexidade do fenômeno de aderência entre o concreto e o PRF, tornam difícil a adoção de um modelo único.

Os modelos para a análise do descolamento encontrados na literatura frequentemente usam a resistência do concreto, o comprimento de ancoragem do PRF, a rigidez axial do PRF, a relação PRF/largura do concreto e a resistência e rigidez do adesivo para prever a aderência entre o PRF e o concreto. A resistência à compressão do concreto provou (Dai et al., 2005; Ko et al., 2014; Nakaba et al., 2001; Savoia et al., 2003) ser um fator crítico na aderência entre o concreto e os sistemas PRF. No entanto, a literatura reporta diferenças consideráveis nas relações entre as resistências médias de aderência e resistências à compressão do concreto, podendo estas serem proporcionais a $\mathrm{fc}^{1 / 2}, \mathrm{fc}^{2 / 3}, \mathrm{fc}^{1 / 5} \mathrm{e} \mathrm{fc}^{0,19}$ para concretos de 20MPa a 50MPa (Chajes et al., 1996; Horiguchi, 1997; Sato et al., 2000; Nakaba et al., 2001). Além disso, o banco de dados experimental disponível na literatura contém poucos dados de resistências à compressão do concreto superiores a 50MPa (Dai et al., 2005; Smith \& Teng, 2002; Ko et al., 2014; Kurtz et al., 2018) e não menciona resistências à compressão superiores a $75 \mathrm{MPa}$. Por outro lado, as expressões utilizadas para determinação do módulo de elasticidade e da resistência à tração do concreto adotadas nos modelos de cálculo existentes estão frequentemente relacionadas à resistência à compressão do concreto.

Neste contexto, considerando que as propriedades mecânicas do concreto afetam a relação de aderência na interface PRF/concreto (Dai et al., 2005; Lu et al., 2005), o uso crescente de concreto de alta resistência na infraestrutura civil exige mais investigações sobre a aderência com sistemas PRF. Sendo assim, este trabalho investiga a influência da resistência à compressão do substrato de concreto no descolamento interfacial de sistemas PRFC laminados in situ. Os resultados são discutidos em termos de deflexões, deformações, fissuras, capacidade de carga final, modo de falha e desempenho de ligação PRF-concreto. 


\section{Programa Experimental}

Os procedimentos experimentais para avaliar a aderência entre sistemas PRF e concreto baseiam-se no estado de tensão da interface, considerando tensões normais (Modo I), tensões axiais (Modo II), ou ambas (Modo III). Os ensaios de tração direta são aplicados para o Modo I, os ensaios de cisalhamento simples ou duplo correspondem ao Modo II (López-González et al., 2012), e os ensaios do tipo viga, em que as tensões normais e de cisalhamento são induzidas na interface PRF/concreto, são usados para o Modo III (LópezGonzález et al., 2012; Wang, 2006).

Este programa experimental se baseia em ensaios de flexão realizados em vigas de concreto armado em escala reduzida, segundo um esquema de ensaio de flexão em quatro pontos: viga simplesmente apoiada com duas cargas verticais espaçadas em $100 \mathrm{~mm}$ aplicadas ao longo do vão de $600 \mathrm{~mm}$ (Figura 2). 0 método de ensaio foi adaptado da norma ASTM D 7958 (ASTM, 2020). As vigas foram armadas com duas barras de aço de $5 \mathrm{~mm}(r=0,0021$, tensão de escoamento $760 \mathrm{MPa}$, deformação de escoamento 3,61 \%o, tensão última $771 \mathrm{MPa}$ e módulo de elasticidade $210 \mathrm{GPa}$ ) e estribos de aço de $6,3 \mathrm{~mm}$ a cada $75 \mathrm{~mm}$.
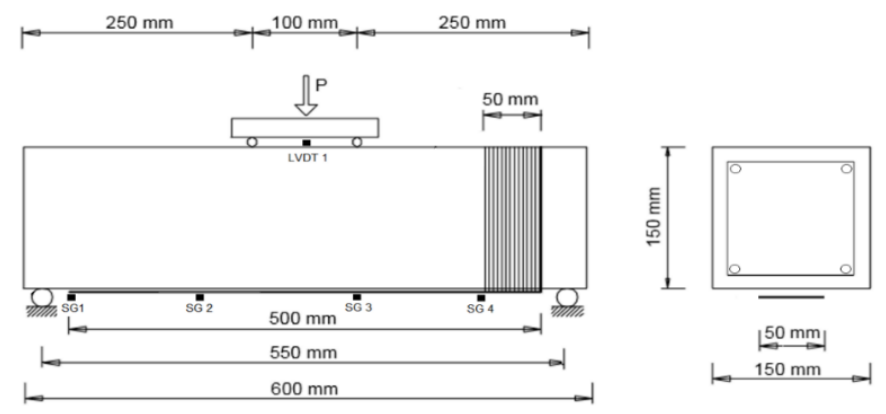

Figura 2 - Esquema de ensaio.

A face inferior das vigas foi reforçada com uma camada de PRFC de 0,167 $\mathrm{mm} \times 50 \mathrm{~mm} \times 500 \mathrm{~mm}$ (espessura $x$ largura $x$ comprimento). Além disso, uma ancoragem em forma de $U$ em uma das extremidades, com 0,167 $\mathrm{mm} \times 50 \mathrm{~mm}$ (espessura $\times$ largura), foi aplicada sobre o reforço, atingindo a altura total da viga. $O$ esquema de reforço foi proposto para induzir o descolamento interfacial a partir da extremidade da viga ou pela propagação de fissuras intermediárias de flexão ou cisalhamento. O sistema PRFC é composto por um tecido unidirecional de fibra de carbono (Fosfiber C N300, resistência à tração $3.550 \mathrm{MPa}$, deformação última 15 $\%$, módulo de elasticidade $235 \mathrm{GPa}$, espessura $0,167 \mathrm{~mm}$ ) e um adesivo epóxi (CF55, resistência à tração 60 $\mathrm{MPa}$, potlife de $25 \mathrm{~min}$ a $25^{\circ} \mathrm{C}$ e $20 \mathrm{~min}$ a $35^{\circ} \mathrm{C}$ ). Foram utilizadas vigas com diferentes resistências à compressão do concreto (Tabela 1), conforme resumido na Tabela 2. Foram testadas 3 amostras de cada série.

Tabela 1 - Dosagem dos concretos.

\begin{tabular}{|l|r|r|r|r|r|r|r|}
\hline Concreto & $\begin{array}{r}\text { Cimento } \\
\mathbf{k g} / \mathbf{m}^{\mathbf{3}}\end{array}$ & $\begin{array}{r}\text { Sílica Ativa } \\
\mathbf{k g} / \mathbf{m}^{\mathbf{3}}\end{array}$ & $\begin{array}{r}\text { Areia } \\
\mathbf{k g} / \mathbf{m}^{\mathbf{3}}\end{array}$ & $\begin{array}{r}\text { Brita } \\
\mathbf{k g} / \mathbf{m}^{\mathbf{3}}\end{array}$ & $\begin{array}{r}\text { Água } \\
\mathbf{1} / \mathbf{m}^{\mathbf{3}}\end{array}$ & $\begin{array}{r}\text { Aditivo } \\
\mathbf{1} / \mathbf{m}^{\mathbf{3}}\end{array}$ & $\begin{array}{r}\text { água/cimento } \\
\text { ágator }\end{array}$ \\
\hline C30* & 292 & - & 811 & 1069 & 190 & 93 & 0,65 \\
\hline C50** & 350 & - & 802 & 1069 & 175 & 111 & 0,50 \\
\hline C80** & 469 & 47 & 686 & 1069 & 160 & 170 & 0,31 \\
\hline C90** & 524 & 53 & 652 & 1104 & 140 & 190 & 0,24 \\
\hline
\end{tabular}

*Mix proportioning method proposed by ACl 211.1 (2002); ** Mix proportioning method proposed by Aïtcin (1998).

Tabela 2 - Descrição do programa experimental.

\begin{tabular}{|c|c|c|c|c|}
\hline Vigas & $\begin{array}{l}A_{\text {aço }} \\
\mathrm{mm}^{2}\end{array}$ & $\begin{array}{l}\text { ApRF } \\
\mathrm{mm}^{2}\end{array}$ & $\begin{array}{c}\mathbf{f}_{\mathrm{c}}^{*} \\
\mathrm{MPa}\end{array}$ & $\begin{array}{l}\mathrm{f}_{\mathrm{t}}^{* *} \\
\mathrm{MPa}\end{array}$ \\
\hline CA30 & \multirow{8}{*}{39,27} & - & \multirow{2}{*}{31,40} & \multirow{2}{*}{5,08} \\
\hline PRF_CA30 & & 8,35 & & \\
\hline CA50 & & - & \multirow{2}{*}{53,40} & \multirow{2}{*}{5,67} \\
\hline PRF_CA50 & & 8,35 & & \\
\hline CA80 & & - & \multirow{2}{*}{79,50} & \multirow{2}{*}{6,35} \\
\hline PRF_CA80 & & 8,35 & & \\
\hline CA90 & & - & \multirow{2}{*}{94,10} & \multirow{2}{*}{10,01} \\
\hline PRF_CA90 & & 8,35 & & \\
\hline
\end{tabular}

Resistência à compressão do substrato de concreto e descolamento interfacial de sistemas PRFC laminados in situ. 
Durante os testes, foram registrados os deslocamentos no centro do vão (LVDT 1), deformações específicas no PRF (SG 1 a 4) e a carga aplicada, através de um sistema de aquisição de dados controlado por computador. A abertura das fissuras foi obtida manualmente por meio de inspeção visual. A avaliação dos dados experimentais foi complementada por imagens de MEV (Microscópio Eletrônico de Varredura Tescan Veja 3 LHM).

A tensão de cisalhamento interfacial média em diferentes seções $\left(\tau_{i}\right)$ foi determinada através da Equação 1 , onde $\varepsilon_{i}$ e $\varepsilon_{i-1}$ são as deformações dos sensores fixados à superfície do PRF nas posições i e i-1, $E_{f}$ é o módulo de elasticidade e $t_{f}$ a espessura do PRF.

$\tau_{i}=\frac{E_{f} t_{f}\left(\varepsilon_{i}-\varepsilon_{i-1}\right)}{\Delta_{x}}$

Equação 1

\section{Análise dos Reultados}

A carga última média das vigas reforçadas aumentou ligeiramente com a resistência do concreto (Figura 3). No entanto, o resultado médio das vigas PRF_CA80 e PRF_CA90 mostram que o aumento da resistência à compressão do concreto não necessariamente resulta em maior capacidade de carga para a viga reforçada. As cargas últimas das vigas PRF_CA30, PRF_CA50 e PRF_CA90 são estatisticamente equivalentes, assim como as de PRF_CA80 e PRF_CA90. As cargas últimas das vigas PRF_CA50, PRF_CA80 e PRF_CA90 aumentaram cerca de $3 \%, 10 \%$ e $6 \%$ quando comparadas a PRF_CA30. Wu \& Hemdan (2005) afirmam que a capacidade de carga de elementos reforçados com PRF é governada pela energia de fratura interfacial. No entanto, a energia de fratura interfacial é frequentemente considerada uma função da resistência à compressão ou tração do concreto (Pan \& Leung, 2009).

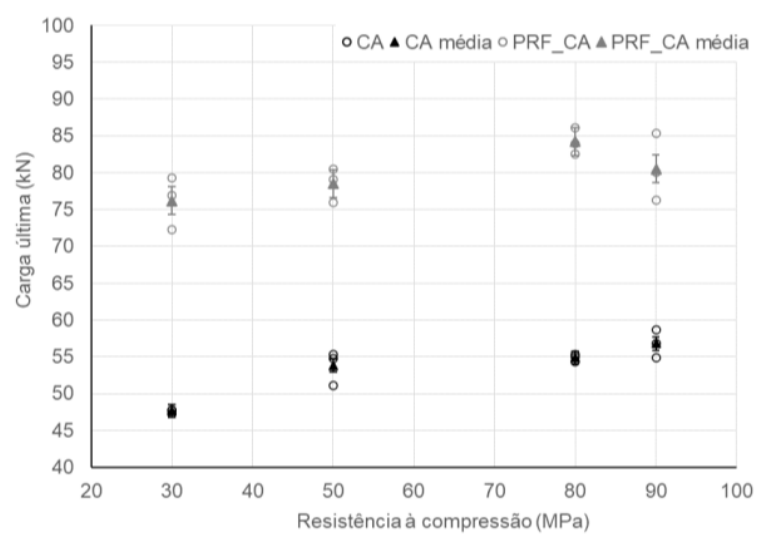

Figura 3 - Cargas últimas versus resistência a compressão co concreto.

O modo de falha de todas as vigas reforçadas está relacionado ao descolamento interfacial devido à propagação de fissura intermediária (Figura 4, Tabela 3). O descolamento do PRF pode ocorrer devido a falha no substrato de concreto, falha na interface do concreto com o adesivo ou do adesivo com o PRF. Uma desvantagem da técnica de moldagem in loco utilizada neste programa experimental é o maior nível de variação que pode ocorrer devido ao processo de fabricação manual (Figura 5), que pode levar ao desalinhamento da fibra e vazios, por exemplo. Tais defeitos de fabricação podem causar falha prematura da matriz devido à concentração de tensões e reduzir o desempenho do reforço (Mehdikhani et al., 2019).

As curvas carga versus deslocamentos para as vigas reforçadas se mostram contínuas nas vigas com concreto de menor resistência à compressão (PRF_CA30 e PRF_CA_50), sem quedas de carga durante o carregamento (Figura 6). Menores resistências à compressão do concreto resultam em menor rigidez e aparecimento mais precoce de microfissuras, contribuindo para uma transferência de carga mais suave para o PRF (Pam \& Leung, 2009). A medida que a resistência à compressão do concreto aumenta, a redistribuição de tensões do concreto para o PRF acarreta as quedas de carga mostradas na Figura 6. Assumindo que a resistência à tração do concreto pode ser estimada em função da resistência à compressão $\left(f_{c t}=0.56 \sqrt{f_{c m}}(\mathrm{ACl}, 2014)\right)$, as curvas apresentadas na Figura 6 corroboram os achados de Niu et al. (2006) e comprovam que a fissuração 
é substancialmente retardada em concretos com alta energia de fratura à tração, cujos efeitos são semelhantes aos das mudanças na resistência à tração do concreto. Como resultado, as quedas de carga ocorrem com carregamentos substancialmente maiores para concretos com alta resistência à tração.

Tabela 3 - Descrição do programa experimental.

\begin{tabular}{|c|c|c|c|c|}
\hline Viga & $\begin{array}{l}\text { Prupt md } \\
\text { (KN) }\end{array}$ & $\begin{array}{l}\text { Pexp PRF_CA / } \\
\text { Pexp CA }^{\text {exp }}\end{array}$ & $\begin{array}{l}\text { Abertura Máxima } \\
\text { de Fissura } \\
\text { (mm) }\end{array}$ & Modo de Falha \\
\hline \multirow{3}{*}{ CA30 } & \multirow{3}{*}{$47,60^{\mathrm{a}}$} & \multirow{3}{*}{-} & 4 & \multirow{3}{*}{ Escoamento da armadura } \\
\hline & & & 4 & \\
\hline & & & 3,5 & \\
\hline \multirow{3}{*}{ PRF_CA30 } & \multirow{3}{*}{$76,85^{1}$} & \multirow{3}{*}{1,61} & 9 & \multirow{3}{*}{$\begin{array}{l}\text { Descolamento interfacial devido a propagação de fissuras } \\
\text { intermediárias de cisalhamento seguido de ruptura do } \\
\text { concreto comprimido }\end{array}$} \\
\hline & & & 5 & \\
\hline & & & 7 & \\
\hline \multirow{3}{*}{ CA50 } & \multirow{3}{*}{$53,75^{b}$} & \multirow{3}{*}{-} & 4 & \multirow{3}{*}{ Escoamento da armadura } \\
\hline & & & 4 & \\
\hline & & & 6 & \\
\hline \multirow{3}{*}{ PRF_CA50 } & \multirow{3}{*}{$78,49^{1}$} & \multirow{3}{*}{1,46} & 2 & \multirow{3}{*}{$\begin{array}{l}\text { Descolamento interfacial devido a propagação de fissuras } \\
\text { intermediárias de cisalhamento/flexão }\end{array}$} \\
\hline & & & 2 & \\
\hline & & & 4 & \\
\hline \multirow{3}{*}{ CA80 } & \multirow{3}{*}{$54,87^{b, c}$} & \multirow{3}{*}{-} & 3,5 & \multirow{3}{*}{ Escoamento da armadura } \\
\hline & & & 3,5 & \\
\hline & & & 3 & \\
\hline \multirow{3}{*}{ PRF_CA80 } & \multirow{3}{*}{$84,21^{2}$} & \multirow{3}{*}{1,53} & 4 & \multirow{3}{*}{$\begin{array}{c}\text { Descolamento interfacial devido a propagação de fissuras } \\
\text { intermediárias de cisalhamento/flexão }\end{array}$} \\
\hline & & & 8 & \\
\hline & & & 5 & \\
\hline \multirow{3}{*}{ CA90 } & \multirow{3}{*}{$56,76^{c}$} & \multirow{3}{*}{-} & 4 & \multirow{3}{*}{ Escoamento da armadura } \\
\hline & & & 4 & \\
\hline & & & 4 & \\
\hline \multirow{3}{*}{ PRF_CA90 } & \multirow{3}{*}{$80,53^{1,2}$} & \multirow{3}{*}{1,42} & 4 & \multirow{3}{*}{$\begin{array}{c}\text { Descolamento interfacial devido a propagação de fissuras } \\
\text { intermediárias de flexão }\end{array}$} \\
\hline & & & 4 & \\
\hline & & & 5 & \\
\hline
\end{tabular}

* Média de 3 amostras, mesmas letras representam médias equivalentes por two-way ANOVA e teste t de Student com $\alpha$ 0,05.

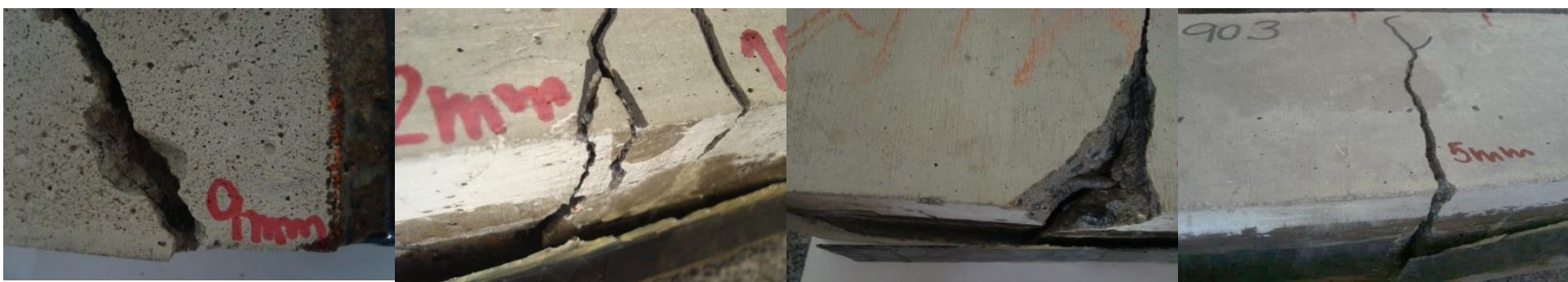

Figura 4 - Modos de falha: (a) Viga PRF_CA30_1; (b) Viga PRF_CA50_1; (c) PRF_CA80_2; (d) PRF_CA90_3.
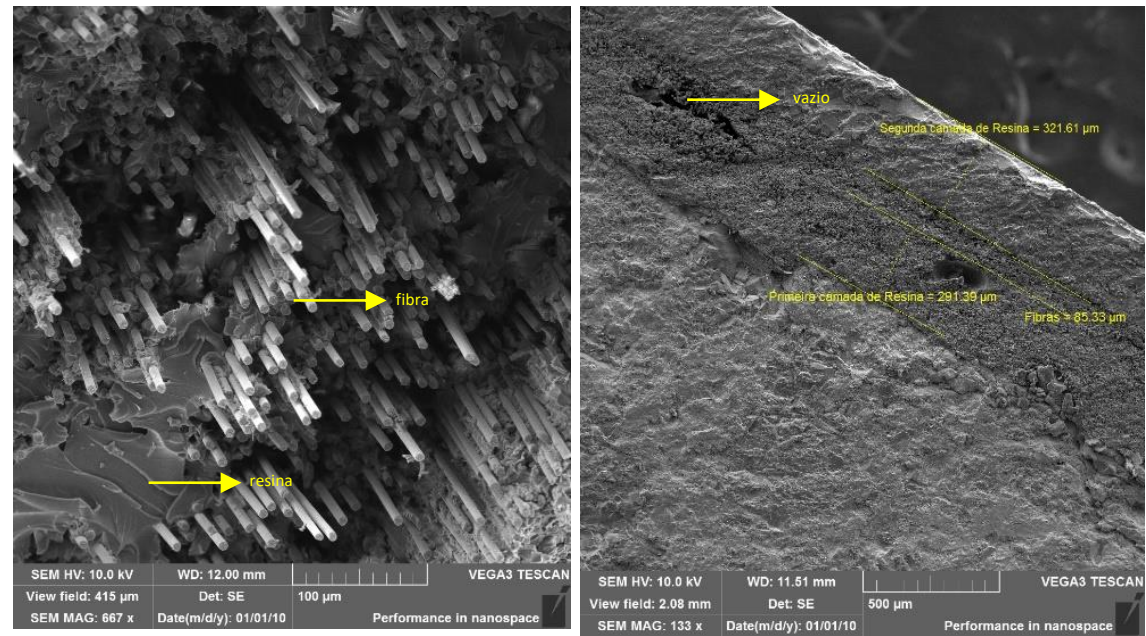

Figura 5 - Variação na composição de PRF moldado in loco (PRF_CA 80).

Resistência à compressão do substrato de concreto e descolamento interfacial de sistemas PRFC laminados in situ. 


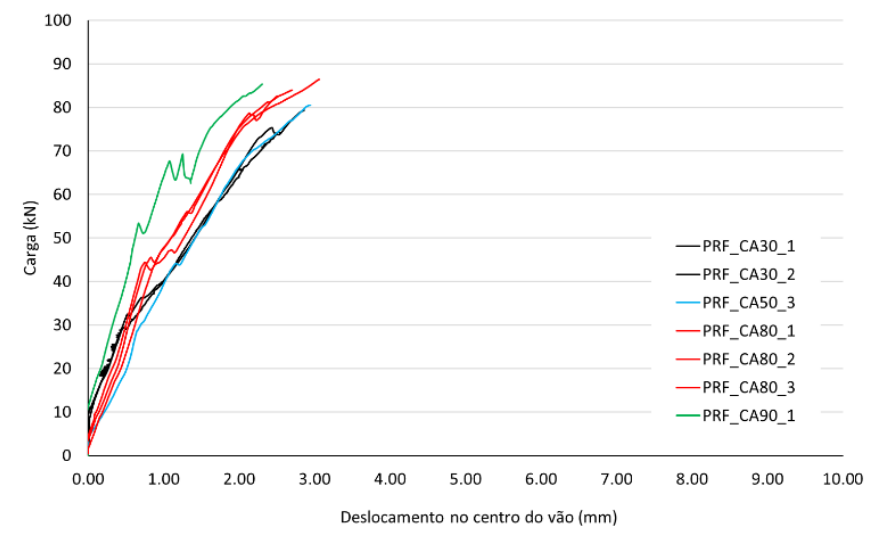

Figura 6 - Deslocamentos no centro do vão: (a) vigas não reforçadas; (b) vigas reforçadas.

Na Figura 7, primeiramente é interessante notar os altos níveis de deformação na ancoragem em forma de $\mathrm{U}$, provavelmente atingidos devido às concentração de tensões na ancoragem. $\mathrm{O}$ uso de laços de PRF uni ou multidirecionais é uma das técnicas de ancoragem mais comuns em sistemas colados externamente. No entanto, se não for executado perfeitamente, podem ocorrer concentração de tensões que induzem falhas prematuras no sistema de ancoragem.

Os perfis de deformação da Figura 7 mostram que, quanto maior a resistência à compressão do concreto, menores são as deformações medidas no SG1. As deformações aumentaram consideravelmente na viga PRF_CA30 após $70 \mathrm{kN}$, o que ocorreu com cargas mais elevadas nas vigas com maior resistência à compressão do concreto. As deformações medidas ao longo das vigas mostram que, para condições de serviço, as deformações são menores nas vigas com maior resistência à compressão do concreto. Para níveis de carga mais elevados, as deformações no PRF ao longo das vigas dependem de outros fatores, como abertura de fissuras e possível concentração de tensões relacionadas a imprecisões no processo de moldagen in loco.
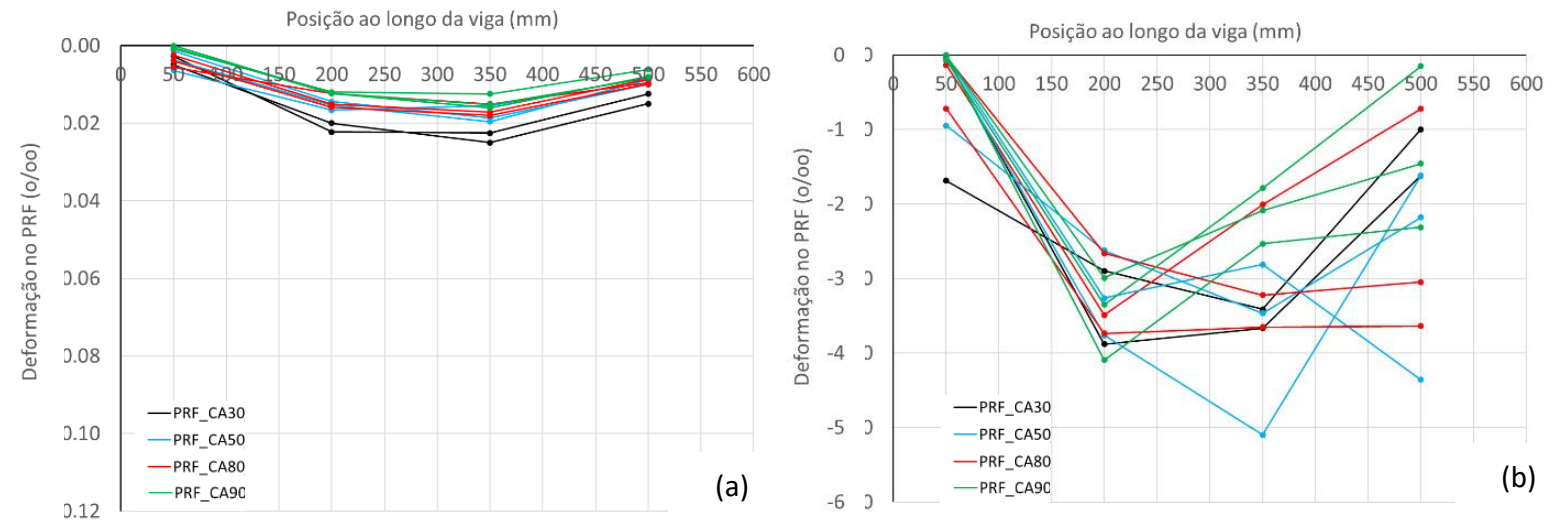

Figura 7 - Deformações específicas no PR: (a) viga submetida a 10kN; (b) na carga de ruptura.

Os resultados plotados no gráfico da Figura 8 mostram que a tensão de cisalhamento interfacial média aumenta quanto maior a carga aplicada e é maior para as vigas da série PRF_CA30. A baixa tensão de cisalhamento interfacial da viga PRF_CA90 resultou nos menores níveis de reforço taxas de transferência de tensões atingidos, e contribuiu também para que o descolameto ocorresse a partir da fissura localizada no meio vão, corroborando com os achados de Niu et al. (2006) para vigas reforçadas com concreto de elevada resistência à compressão. Esses resultados, comparados à resposta carga versus deslocamento das vigas reforçadas (Figura 6), comprovaram que o uso de alta resistência à compressão do concreto afetou a resposta de pré-fissuração, a transferência de tensões e a ductilidade geral na ruptura, resultando em um esquema de reforço menos eficiente. De fato, considerando os resultados médios da carga de ruptura das vigas reforçadas (série PRF_CA) e respectivas não reforçadas (série CA), PRF_CA30 e PRF_CA90 apresentaram as maiores $(1,61)$ e menores $(1,42)$ taxas de reforço efetivas (Tabela 3 ). 


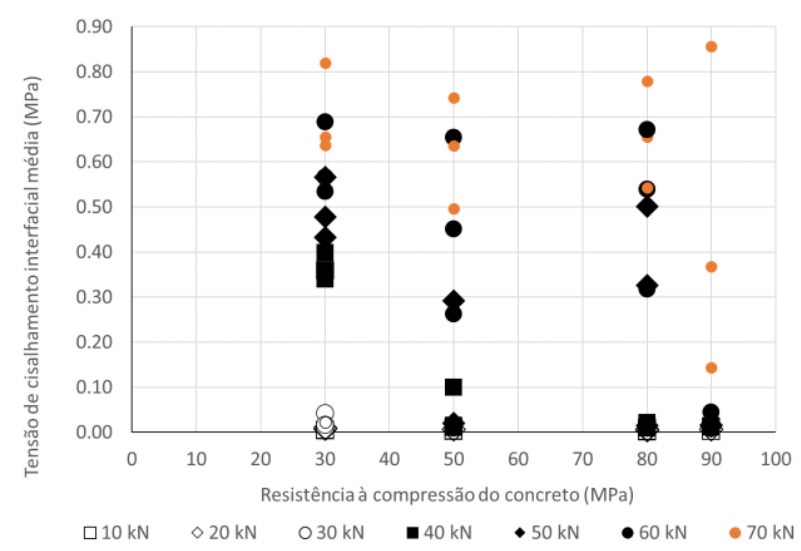

Figura 8 - Tensão de cisalhamento interfacial média versus resistência à compressão do concreto.

Os resultados mostram que a falha de descolamento interfacial PRF é altamente influenciada pela relação entre a fissuração do concreto e as propriedades da interface PRF-concreto. Esta relação está diretamente relacionada às propriedades mecânicas do concreto e PRF (resistência máxima e rigidez, por exemplo), distribuição de tensões sob carga e redistribuição de tensões no estágio pós-fissuração, que dependem das propriedades do concreto e da carga suportrada pela armadura de aço.

A rigidez do PRF é essencial ao considerar a possibilidade de executar um reforço com PRF. Camadas finas de PRF podem resultar em descolamento interfacial devido a fissuras intermediárias, em vez de arrancamento do concreto ou descolamento interfacial a partir da extremidade da viga. Neste programa experimental, as vigas foram reforçadas com uma única camada de PRF e romperam por descolamento interfacial devido à propagação de fissuras intermediárias. Camadas mais espessas de PRF, por outro lado, reduzem a ductilidade do elemento reforçado (Garcez et al., 2012) e levam à ruptura devido ao arrancamento do concreto ou descolamento interfacial devido à propagação de fissura de extremidade.

\section{Conclusões}

As vigas reforçadas romperam por descolamento interfacial devido à propagação de fissuras intermediárias. A ruptura ocorreu a partir da borda de fissuras de flexão/cisalhamento nos substratos de concreto com menor resistência à compressão ou a partir da borda das fissuras de flexão, localizadas no centro do vão, para substratos de concreto com maior resistência à compressão.

O aumento da resistência à compressão do concreto não resultou em aumento de carga última nas vigas reforçadas. Para condições de serviço, as deformações no PRF foram menores quanto maior a resistência à compressão do concreto.

Os mecanismos de iniciação do dano e propagação do descolamento mostraram-se altamente dependentes da transferência de carga e da redistribuição de tensões após a fissuração do concreto.

O uso de alta resistência à compressão do concreto afetou a resposta pré-fissuração, a transferência de tensões e a ductilidade geral dos elementos, resultando em um esquema de reforço de PRF menos eficaz.

\section{Referências Bibliográficas}

ACl Committee 318. Building Code Requirements for Structural Concrete (ACI 318R-14). Farmington Hills (MI); 2014.

$\mathrm{ACl}$ Committee 440. Guide for the Design and Construction of Externally Bonded FRP Systems for Strengthening Concrete Structures (ACl 440.2R-17). Farmington Hills (MI); 2017.

ACl. Standard Practice for Selecting Proportions for Normal, Heavyweight, and Mass Concrete (ACI 211.1-91).

Farmington Hills (MI); 2002.

Aïtcin P-C. High Performance Concrete. 1st ed. London: E \& FN Spon; 1998. 
ASTM. Standard Test Method for Evaluation of Performance for FRP Composite Bonded to Concrete Substrate using Beam Test. West Conshohocken, Pennsylvania; 2020.

Chajes, M J; Finch, W W; Januszka, T F; Thomson TA. Bond and Force Transfer of Composite-Material Plates Bonded to Concrete. ACI Struct J. 1996; 93: 209-17.

Dai J, Ueda T, Sato Y. Development of the Nonlinear Bond Stress-Slip Model of Fiber Reinforced Plastics Sheet-Concrete Interfaces with a Simple Method. J Compos Constr. 2005; 9: 52-62.

Garcez M, Meneghetti L, Silva Filho LCP. Structural performance of RC beams poststrengthened with carbon, aramid, and glass FRP systems. J Compos Constr. 2008; 12: 522-30.

Garcez MR, Filho LCPS, Meier U. Post-strengthening of reinforced concrete beams with prestressed CFRP strips: part 2: analysis under cyclic loading. Rev IBRACON Estruturas e Mater. 2012; 5(4): 420-439.

Horiguchi T. Effect of test methods and quality of concrete on bond strength of CFRP sheet. Int Symp NonMetallic Reinf Concr Struct. 1997. p. 265-70.

Ko H, Matthys S, Palmieri A, Sato Y. Development of a simplified bond stress-slip model for bonded FRPconcrete interfaces. Constr Build Mater. 2014; 68: 142-57.

Kurtz S, Balaguru P, Helm J. Experimental study of interfacial shear stresses in FRP-strengthened RC beams. J Compos Constr. 2008; 12: 312-22.

López-gonzález JC, Fernández-gómez J, González-valle E. Effect of Adhesive Thickness and Concrete Strength on FRP-Concrete Bonds. J Compos Constr. 2012; 16: 705-11.

Lu XZ, Teng JG, Ye LP, Jiang JJ. Bond - slip models for FRP sheets / plates bonded to concrete. Eng Struct. 2005; 27: 920-37.

Mehdikhani M, Gorbatikh L, Verpoest I, Lomov S V. Voids in fiber-reinforced polymer composites: A review on their formation, characteristics, and effects on mechanical performance. J Compos Mater. 2019; 53: 1579-669.

Nakaba K, Kanakubo T, Furuta T, Yoshizawa H. Bond behavior between fiber-reinforced polymer laminates and concrete. ACl Struct J. 2001; 98: 359-67.

Niu H, Karbhari VM, Wu Z. Diagonal macro-crack induced debonding mechanisms in FRP rehabilitated concrete. Compos Part B Eng. 2006; 37: 627-41.

Pan J, Leung CKY. Effect of concrete composition on interfacial parameters governing FRP debonding from the concrete substrate. Adv Struct Eng. 2009; 12: 627-37.

Sato, Y; Asano, Y; Ueda T. Fundamental study on bond mechanism of carbon fiber sheet. J Civ Eng Stud. 2000;648:97-115.

Savoia, M; Farracuti, B; Mazzotti D. Non-linear bond-slip law for FRP-concrete interface. Proc 6th Int Symp FRP Reinf Concr Struct. Singapore: World Scientific Publications; 2003. p. 163-72.

Siddika A, Al Mamun MA, Ferdous W, Alyousef R. Performances, challenges, and opportunities in strengthening reinforced concrete structures by using FRPs - A state-of-the-art review. Eng Fail Anal. 2020;111.

Smith ST, Teng JG. FRP-strengthened RC beams. I: review of debonding strength models. Eng Struct. 2002; 24: 385-95.

Triantafillou T, Matthys S. Fibre-reinforced polymer reinforcement enters fib Model Code 2010. Struct Concr. 2013; 14: 335-41.

Ulaga T, Vogel T, Meier U. Bilinear Stress-Slip Bond Model: Theoretical Background and Significance. Proceeding FRPRCS-6 Conf. Singapore; 2003. p. 153-62.

Wang J. Cohesive zone model of intermediate crack-induced debonding of FRP-plated concrete beam. Int J Solids Struct. 2006; 43: 6630-48.

Wu Z, Hemdan S. Debonding in FRP-Strengthened Flexural Members with Different Shear-Span Ratios. Proc 7th Int Symp Fiber- Reinf Polym Reinf Concr Struct. 2005. p. 411-26. 